\title{
Shock tube experiments and Fe-simulation of the structural and material non-linear transient response of plates subjected to blast loading
}

\author{
R. Schmidt, M. Stoffel \& T. D. Vu \\ RWTH Aachen University, Germany
}

\begin{abstract}
This paper deals with the experimental investigation, modelling, and finite element simulation of structures exposed to shock-type blast loading conditions taking into account the structural and material non-linear effects. First- and thirdorder transverse shear deformation theories of plates and shells serve as basis of a finite element algorithm for the simulation of the transient, geometrically nonlinear elastic-viscoplastic response. Isoparametric Lagrangian 9-node shell finite elements and the central difference method for the time integration of the nonlinear equations of motion are used. Experiments are performed on thin clamped circular aluminium and steel plates in shock tubes. The main advantage of this experimental technique is that the front wave impinging on the structure is plane and yields a uniformly distributed pressure pulse. Consequently, in contrast to other experimental methods reported in literature, the time history of the shocktype loading can be modelled easily during the FE analysis. Comparative numerical simulations using first- or third-order transverse shear deformation plate theory, respectively, show a very good agreement with the experimental results. The best correlation with the experimentally observed transient response and permanent deflection is obtained by the refined, third-order transverse shear deformation model.
\end{abstract}

Keywords: blast loading, shock tube, first- and third-order transverse shear deformation theory, viscoplasticity.

\section{Introduction}

The present paper deals with modelling, computational simulation and experimental investigation of the transient large deflection elastic-viscoplastic 
response of plates and shells subjected to impulsive loading. The focus of the paper is on comparative non-linear FE simulation, based on either first- or third order transverse shear deformation plate and shell theory.

The theoretical and numerical developments are based on geometrically nonlinear plate and shell theories and associated finite element models available in our earlier papers (Schmidt and Reddy [1], Palmerio et al. [2], Vu et al. [3]). Experimental investigations of blast loaded plates and comparative numerical simulations of the transient response have been reported already in our papers [46], where a very good agreement was achieved by using FE models based on first-order transverse shear deformation theories. Our results showed, however, that the constant transverse shear stress assumption, implicit in first-order transverse shear deformation theories, leads to local over- and underprediction, respectively, of the equivalent plastic strain rates through the thickness that can affect the accuracy of the predicted transient response and permanent deflections. Therefore, in the present paper the third-order transverse shear deformation model is used for comparative FE analysis.

The theoretical predictions of the time history responses and final permanent structural deformations are compared with results of experimental tests performed on thin aluminium and steel plates in shock tubes. By means of this experimental device impulsive surface loads with various intensities and time histories can be applied resulting in geometrically non-linear elastic-viscoplastic vibrations. Experimental investigations of structures subjected to blast loading conditions are frequently performed by shock waves generated by explosives (e.g. gaseous mixtures, charges of plastic explosives) that detonate at some distance of the structure (see Idczak et al. [7, 8], Renard and Pennetier [9], Pennetier [10], among others). These experimental techniques lead to a complex non-uniform time-space evolution of the pressure distribution on plane structural elements thus requiring a sophisticated modelling and simulation of the blast loading conditions. The main advantage of the shock tube technique used in this paper is that the shock wave front is plane, thus yielding a uniformly distributed pressure pulse on plates which can be measured easily by only one pressure transducer integrated in the specimen mounting ring. This allows for precise modelling of the transient loading conditions during simulations of the experimentally observed plate response.

The topics addressed in this presentation include (a) the correlation of experimental and simulated transient inelastic response and permanent deflections, (b) the effect of the transverse shear stress distribution (first-order versus third-order transverse shear deformation hypothesis) on the local evolution of the material behaviour and on the global dynamic response, (c) the evolution of deflections, stresses, and plastic zones under blast loading conditions. A good agreement between the computational and experimental results for the elastic-viscoplastic plate response is observed. It turns out that the best correlation is obtained by means of the third-order transverse shear deformation theory. Special emphasis is focused on the evolution of bending moments and equivalent plastic strain rates, where considerable differences can 
be observed when first- or third-order transverse shear deformation theory, respectively, is applied.

\section{Experimental setup}

For the experiments shock tubes (diameter $458 \mathrm{~mm}$ and $108 \mathrm{~mm}$, respectively) are used in order to subject clamped circular aluminium and steel plates (thickness $2 \mathrm{~mm}$, diameter $553 \mathrm{~mm}$ and $138 \mathrm{~mm}$, respectively) to impulsive loadings, see [6]. The high pressure chamber (HPC) is separated from the low pressure chamber (LPC) by a diaphragm. The pressure in the HPC is increased until the diaphragm is ruptured. A shock wave travels through the LPC and impinges on the plate specimen clamped at the end of the tube between two ring flanges leading to a plane high-pressure pulse. The time histories of the centre deflection and of the pressure acting on the plate are recorded by a capacitor and a piezoelectric pressure sensor, respectively.

\section{Structural modelling}

Consider a plate or shell with volume $V$, total bounding surface $B$, mid-surface $M$ and thickness $h$ subjected to blast loading conditions. The principle of virtual displacements of 3-D elastokinetics reads

$$
\int_{\mathrm{V}}\left\{s^{i j} \delta E_{i j}-\rho\left(B^{i}-A^{i}\right) \delta V_{i}\right\} d V-\int_{\mathrm{B}}\left({ }^{*} s^{i}+D^{i}\right) \delta V_{i} d B=0,
$$

where $V_{i}$ denote the covariant components of the displacement vector in an arbitrary point of the body, $s^{i j}$ and $E_{i j}$ are the components of the second PiolaKirchhoff stress and Green strain tensor, $\rho$ is the density of the undeformed body, and $B^{i}, A^{i}$ are components of the body force and acceleration vectors per unit volume of the undeformed body, respectively. Furthermore, ${ }^{*} s^{i}$ and $D^{i}$ are components of the prescribed external stress vector and of the damping force vector, respectively, per unit area of the undeformed shell boundary surface $B$. For viscous damping the damping force vector components may be written as $D^{i}=-D^{i j} \dot{V}_{j}$ with $D^{i j}$ denoting the components of the damping tensor. Here and throughout the paper the Einstein summation convention is used with Latin indices ranging from 1 to 3 and Greek indices ranging from 1 to 2 . The displacement vector $V$ of any point in the shell space can be referred to the contravariant base vector triad of the reference surface as

$$
\underset{\sim}{V}=v_{\alpha} \underset{\sim}{a}+v_{3} \underset{\sim}{n}
$$

Refined non-linear first-order transverse shear deformation shell theories (FOSD), which account for small strains (of $O(\eta), \eta<<1$ ), small rotations (of $O(\eta)$ ) about the normal, and moderate rotations (of $O\left(\eta^{1 / 2}\right)$ ) of the normal, have been derived by Schmidt and Reddy [1]. They are based on the hypothesis of a linearly varying displacement field through the thickness, i.e. 


$$
v_{\alpha}=\stackrel{(0)}{v_{\alpha}}+\theta^{3} \stackrel{(1)}{v_{\alpha},} \quad v_{3}=\stackrel{(0)}{v_{3}},
$$
where $v_{\alpha}$ and $v_{3}$ denote the tangential and normal displacement components at the reference surface, $v_{\alpha}$ stands for the rotations of the normal and $\theta^{3}$ is the distance from the reference surface. Based on the above assumptions the Green strain tensor components can be approximated, to within a negligible small relative error margin (of $O(\eta)$ ), by the series expansions

$$
E_{\alpha \beta}=\stackrel{(0)}{E}_{\alpha \beta}+\theta^{3} \stackrel{(1)}{E_{\alpha \beta}}+\left(\theta^{3}\right)^{2} \stackrel{(2)}{E_{\alpha \beta}}, \quad E_{\alpha 3}=\stackrel{(0)}{E_{\alpha 3}}+\theta^{3} \stackrel{(1)}{E_{\alpha 3}}, \quad E_{33}=\stackrel{(0)}{E_{33}} .
$$

The non-linear moderate rotation FOSD shell strain-displacement relations of Schmidt and Reddy [1] specified for the kinematical hypothesis (3) with all transverse normal strain effects neglected yield the following five-parameter variant of the 2-D strain measures

$$
\begin{aligned}
& \stackrel{(0)}{E_{\alpha \beta}}=\stackrel{(0)}{\theta}_{\alpha \beta}+\frac{1}{2} \stackrel{(0)}{\varphi}_{\alpha} \stackrel{(0)}{\varphi}_{\beta},
\end{aligned}
$$

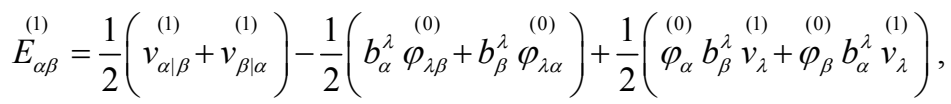

$$
\begin{aligned}
& E_{\alpha \beta}^{(2)}=-\frac{1}{2}\left(b_{\alpha}^{\lambda v_{\lambda \mid \beta}^{(1)}}+b_{\beta}^{\lambda} v_{\lambda \mid \alpha}^{(1)}\right)+\frac{1}{2} b_{\alpha}^{\lambda} b_{\beta}^{\kappa} v_{\lambda}^{(1)} v_{\kappa}^{(1)} \\
& \stackrel{(0)}{E_{\alpha 3}}=\frac{1}{2}\left(\begin{array}{c}
(0) \\
\varphi_{\alpha}
\end{array}+{\stackrel{(1)}{v_{\alpha}}}^{\prime}\right)+\frac{1}{2} v^{(1)} \stackrel{(0)}{\varphi}_{\lambda \alpha}, \quad \stackrel{(1)}{E_{\alpha 3}}=\frac{1}{2} v^{(1)}{ }^{(1)} v_{\lambda \mid \alpha}, \quad \stackrel{(0)}{E}_{33}=0 .
\end{aligned}
$$

Here the following abbreviations have been used:

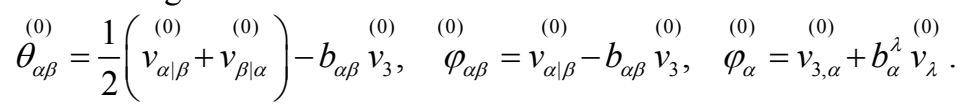

The notations (.), $\alpha$ and (.) $)_{\alpha}$ stand for partial and covariant differentiation on the reference surface and $b_{\alpha \beta}, b_{\alpha}^{\lambda}$ denote the covariant and mixed components of the curvature tensor. For zero curvature the equations above yield the von Kármán-type non-linear FOSD plate theory.

The results referring to the third-order shear deformation theory (TOSD) are obtained with a finite plate element developed by $\mathrm{Vu}$ et al. [3]. The finite element is based on the von Kármán-type non-linearity, too. The TOSD hypothesis for the through-thickness variation of the tangential and normal displacement components reads

$$
v_{\alpha}=\stackrel{0}{v_{\alpha}}+\theta^{3} \stackrel{1}{v_{\alpha}}+\left(\theta^{3}\right)^{2} \stackrel{2}{v_{\alpha}}+\left(\theta^{3}\right)^{3} \stackrel{3}{v_{\alpha}}, \quad v_{3}=\stackrel{0}{v_{3}},
$$

where the constant, linear, quadratic and cubic terms are denoted by $0,1,2$ and 3 , respectively. The above assumption for the tangential and normal displacement field yields 9 degrees of freedom per nodal point. In order to reduce the degrees of freedom from 9 to 5 , it is assumed that no shear traction is applied on the bottom $\left(\theta^{3}=-h / 2\right)$ and on the top surface $\left(\theta^{3}=h / 2\right)$ of the plate. For plates of isotropic material or laminated of orthotropic layers this is equivalent to 


$$
E_{\alpha 3}\left(\theta^{3}=\frac{h}{2}\right)=0, \quad E_{\alpha 3}\left(\theta^{3}=-\frac{h}{2}\right)=0,
$$

which yields

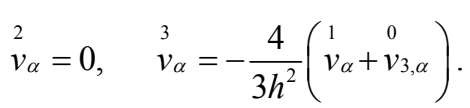

Taking all assumptions into consideration, the Green-Lagrange strain components for the von Kármán-type non-linearity TOSD plate element can be expressed as:

$$
E_{\alpha \beta}=\stackrel{0}{E}_{\alpha \beta}+\Theta^{3} \stackrel{1}{E}_{\alpha \beta}+\left(\Theta^{3}\right)^{3} \stackrel{3}{E}_{\alpha \beta}, \quad E_{\alpha 3}=\stackrel{0}{E}_{\alpha 3}+\left(\Theta^{3}\right)^{2} \stackrel{2}{E}_{\alpha 3}, \quad E_{33}=0,
$$

where

$$
\begin{aligned}
& \stackrel{0}{E}_{\alpha \beta}=\frac{1}{2}\left(\begin{array}{cccc}
0 & 0 & 0 & 0 \\
v_{\alpha \mid \beta}+v_{\beta \mid \alpha} & \underline{v_{3, \alpha}} v_{3, \beta}
\end{array}\right), \quad \stackrel{1}{E}_{\alpha \beta}=\frac{1}{2}\left(\begin{array}{ll}
1 & 1 \\
v_{\alpha \mid \beta} & +v_{\beta \mid \alpha}
\end{array}\right), \\
& \stackrel{3}{E_{\alpha \beta}}=-\frac{2}{3 h^{2}}\left(\begin{array}{cc}
1 & 1 \\
v_{\alpha \mid \beta} & +v_{\beta \mid \alpha}+2 \\
v_{3 \mid \alpha \beta}
\end{array}\right),
\end{aligned}
$$

and

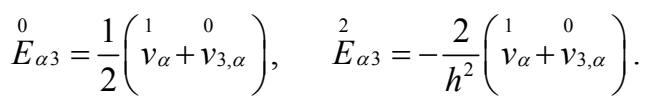

The cubic term of the tangential strains is neglected in the numerical applications. This can be justified for thin-walled structures.

The transformation of the principle of virtual displacements of 3-D elastokinetics Eq. (1) into a 2-D formulation yields

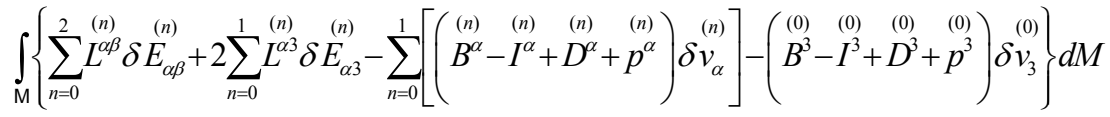

$$
\begin{aligned}
& -\int_{C}\left\{\sum_{n=0}^{1}\left[{ }^{*} L^{\alpha \beta} v_{\alpha} v_{\beta} \delta v_{v}^{(n)}+{ }^{*} L^{\alpha \beta} t_{\alpha} v_{\beta} \delta v_{t}^{(n)}\right]+{ }^{*} L^{3 \beta} v_{\beta} \delta v_{3}^{(0)}\right\} d C=0 .
\end{aligned}
$$

Here, $\stackrel{(n)}{L}^{i j}$ are the gross stress resultants, $\stackrel{(n)}{B^{i}}, \stackrel{(n)}{I^{i}}$, and $\stackrel{(n)}{D^{i}}$ denote the gross body, inertia and damping couples, while $\stackrel{(n)}{p^{i}}$, and ${ }^{*} L^{i j}$ are the gross surface and boundary load couples, respectively, of the n-th order. They are obtained by integration of the respective physical quantities through all layers and can be found in detail in Librescu and Schmidt [11], Schmidt and Reddy [1]. Furthermore, in Eq. (13) $C$ denotes the boundary curve of the reference surface $M, v_{\alpha}$ and $t_{\alpha}$ are the components of the unit outward normal and tangent vector of $C$, while indices $v$ and $t$ denote normal and tangential displacement and rotation components, respectively.

For viscoplastic transient analysis the constitutive equations of Chaboche [12] are employed. Details of the material parameter identification by uniaxial tension tests are available in Stoffel et al. [4]. A layered shell model is used that permits to trace the evolution of the material properties in each individual layer. Numerical solutions are obtained by using the isoparametric Lagrangian 9-node 
shell finite element and the central difference method for the time integration of the non-linear equations of motion (see $[2,13,14]$ ).

\section{Results}

Fig. 1 shows the evolution of the pressure and centre deflection, respectively, recorded during a typical shock tube experiment using aluminium plate specimens (thickness $2 \mathrm{~mm}$, diameter $553 \mathrm{~mm}$ ) as described in Chapter 2. One can observe a very good agreement between the results predicted by $\mathrm{FE}$ simulation of the elastic-viscoplastic transient response and the experimental observation. The results obtained by TOSD theory mach the experimental results closer than those obtained by FOSD theory.

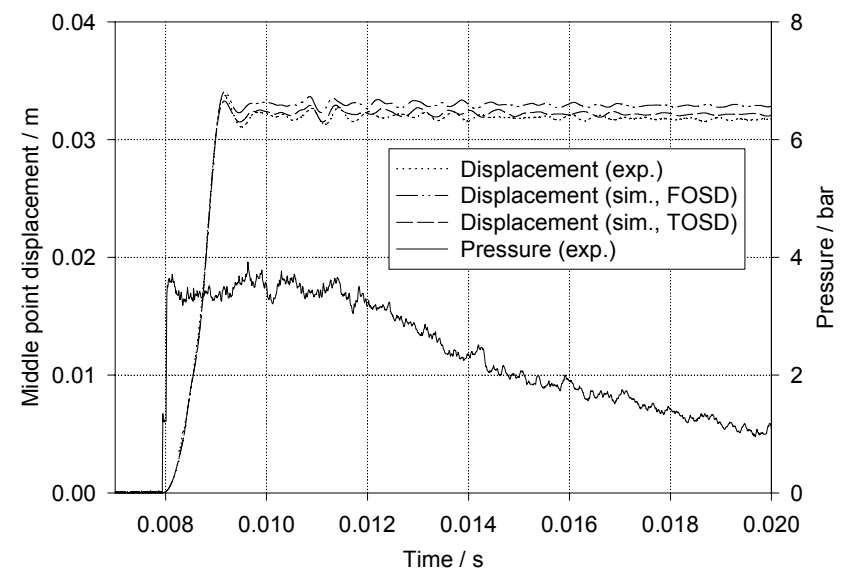

Figure 1: Time history of pressure and centre displacement.

In the above experiment the peak pressure during blast loading was 3.5 bar. Fig. 2 shows FE simulation results for the evolution of the bending moment at the plate centre and at the plate boundary.

It can be observed from Fig. 2 that immediately after the arrival of the shock wave high bending moments occur only close to the boundary. Then the bending action spreads inwards and reaches the plate centre with a delay. Note also that in the plate centre oscillations of the bending moment occur. First small oscillations are visible, than the amplitudes increase, and finally the bending moment tends to zero. This indicates that the movement of the centre during the deformation is much more complicated than it is suggested by Fig. 1. In addition to the bending moment a rapid increase of the membrane force occurs almost simultaneously in all points. This reflects the von Kármán effect and indicates that the blast loading problem turns into a geometrically non-linear one already immediately after the arrival of the shock wave.

The above observations can be explained by flexural waves that originate at the boundary and travel towards the plate centre at different speeds. In the 
present case the impulsive transversal loading of the boundary, which occurs as reaction to the pressure impulse on the plate, generates such flexural waves with a wide spectrum of frequencies. According to Doyle [15] the propagation velocity of flexural waves increases with increasing frequency. Furthermore, spectral analysis of the investigated plate vibration indicates that wave amplitudes decrease with increasing frequency. Consequently, those waves with high frequency and small amplitude reach the plate centre first. This results in small amplitude bending oscillations in the plate centre as can be observed in Fig. 2. With a considerable delay the flexural waves with the highest amplitude and lowest frequency reach the plate centre at last what can be seen in Fig. 2, too. At the boundary these oscillations do not occur, because the waves originate there all at the same instant of time.

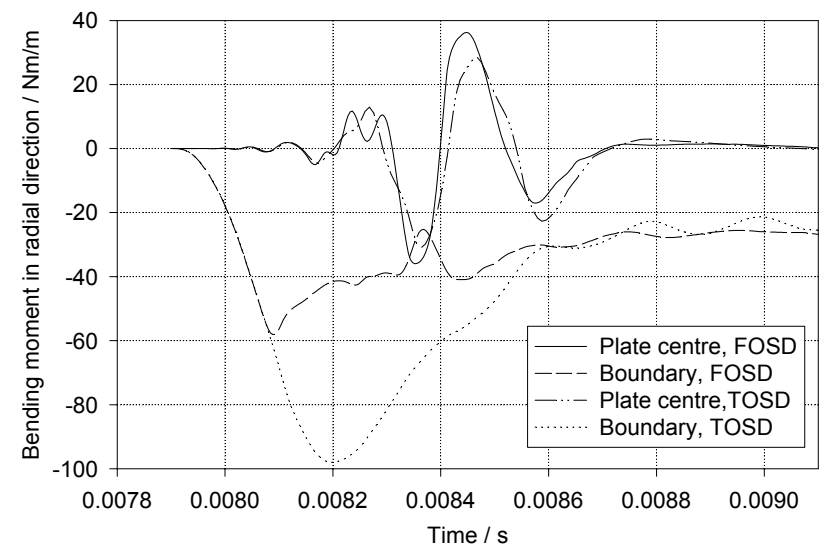

Figure 2: Evolution of the bending moment.

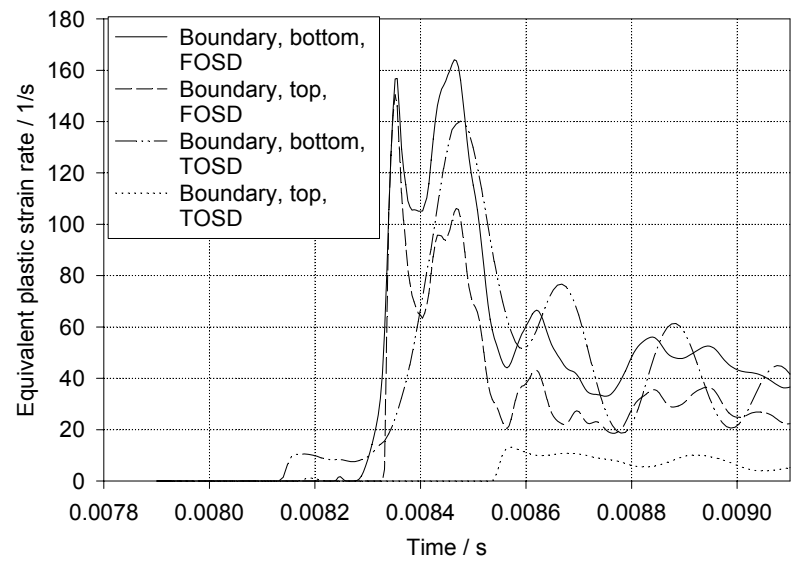

Figure 3: Evolution of the equivalent plastic strain rate at the boundary. 
The above observations are also reflected in the evolution of the equivalent plastic strain rate shown in Fig. 3. At the boundary the negative moment causes stretching at the bottom of the plate. Together with the action of the tensile membrane forces and shear forces plastic zones develop first at the boundary bottom layer. It is interesting to note that for both the top and bottom layer the FOSD theory predicts higher equivalent plastic strain rates than the TOSD theory, in time intervals where the predicted bending moments are almost equal. This is due to the fact that according to the FOSD hypothesis the transverse shear stress distribution is constant through the thickness, while in the TOSD theory it is parabolic and tends to zero (see Eq. (8)) at the bounding surfaces.

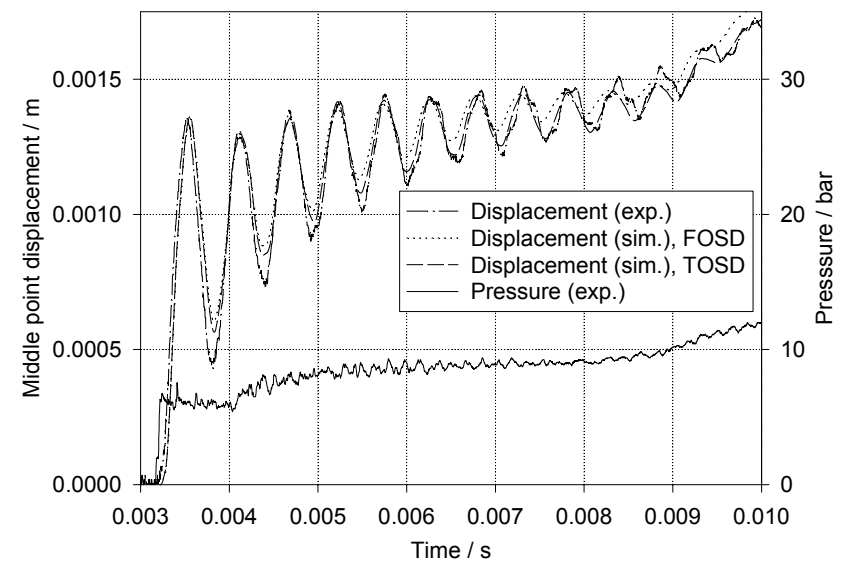

Figure 4: Time history of pressure and centre displacement.

Fig. 4 shows the evolution of the pressure and centre deflection, respectively, recorded during a typical shock tube experiment using steel plate specimens (thickness $2 \mathrm{~mm}$, diameter $138 \mathrm{~mm}$ ) as described in Chapter 2. Also here one can observe a very good agreement between the results predicted by FE simulation of the elastic-viscoplastic transient response and the experiment. Again it can be noticed that the TOSD theory performs better than the FOSD theory, especially for increasing time. Hence, also the permanent deformations of the structure are predicted more accurately by the TOSD theory.

\section{Conclusions}

The results presented in this paper demonstrate that simulation of blast loaded structures cannot be based on bending or non-linear membrane theories but require models which take into account the complicated membrane-bending interaction already during the impulse duration. Furthermore, the results of the present investigations show clearly that the constant transverse shear stress assumption, implicit in first-order transverse shear deformation theories, leads to local over- and underprediction, respectively, of the equivalent plastic strain rates 
through the thickness, depending on the distance of the point under consideration from the mid-surface. This can affect the accuracy of the predicted transient response and permanent deflections. Therefore, in the present paper the results of simulations based on third-order transverse shear deformation theory showed a better correlation with the experimental results.

\section{References}

[1] Schmidt, R., Reddy, J.N. : A refined small strain moderate rotation theory of elastic anisotropic shells, ASME J. Appl. Mech. 55 (1988), 611-617.

[2] Palmerio, A.F., Reddy, J.N., Schmidt, R. : On a moderate rotation theory of laminated anisotropic shells, Part 1: Theory, Part 2: Finite element analysis, Int. J. Non-Linear Mech. 25 (1990), 687-714.

[3] Vu, T.D., Lentzen, S., Schmidt, R. : Geometrically nonlinear FE-analysis of piezolaminated plates based on first- and third-order shear deformation theory, Proceedings of the 8th International Conference on Mechatronics Technology, ICMT 2004, Hanoi, Vietnam, 8 - 12 November 2004, eds. Nguyen Khoa Son, Pham Thuong Cat, Pham Anh Tuan, 267-272, Vietnam National University Publisher, Hanoi 2004.

[4] Stoffel, M., Schmidt, R., Weichert, D. : Shock wave-loaded plates, Int. J. Sol. Struc. 38 (2001), 7659-7680.

[5] Stoffel, M., Schmidt, R., Weichert, D. : Simulation and experimental validation of the dynamic response of viscoplastic plates, in "Computational Methods and Experimental Measurements X", 505-514, WIT Press, Southampton 2001.

[6] Schmidt, R., Stoffel, M. : Modelling, Simulation and experimental investigation of plates subjected to blast loading conditions, in: "Computational Ballistics II", eds. V. Sanchez-Galvez, C.A. Brebbia, A.A. Motta, G.E. Anderson, 249-258, WIT Press, Southampton-Boston 2005.

[7] Idczak, W., Rymarz, Cz., Spychala, A. : Large deflection of a rigidviscoplastic impulsively loaded circular plate, J. Tech. Phys. 21 (1980), 473-487.

[8] Idczak, W., Rymarz, Cz., Spychala, A. : Studies on shock-wave loaded, clamped circular plates, J. Tech. Phys. 22 (1981), 175-184.

[9] Renard, J., Pennetier, O. Nonlinear dynamic response of plates submitted to an explosion - Numerical and experimental study, Structural Dynamics Eurodyn `96, 689-694, Balkema, Rotterdam (1996).

[10] Pennetier, O. : Interaction Structures-Detonations, Thesis, Université d'Orleans 1998.

[11] Librescu, L., Schmidt, R. : Refined theories of elastic anisotropic shells accounting for small strains and moderate rotations, Int. J. Non-Linear Mech. 23 (1988), 217-229.

[12] Chaboche, J.L. : Constitutive equations for cyclic plasticity and cyclic viscoplasticity, Int. J. of Plast. 5 (1989), 247-302.

[13] Klosowski, P., Schmidt, R. : Geometrically and physically nonlinear transient analysis of structures, in "Dynamical Problems in Mechanical 
Systems IV", 171-182, eds. R. Bogacz, G.P. Ostermeyer, K. Popp, Polish Academy of Sciences, Warsaw 1996.

[14] Kreja, I., Schmidt, R., Reddy, J.N. : Finite elements based on a first-order shear deformation moderate rotation shell theory with application to the analysis of composite structures, Int. J. Non-Linear Mech. 32 (1997), 11231142.

[15] Doyle, J.F. : Wave propagation in structures, Springer Verlag, BerlinHeidelbeg-New York, 1997. 